\title{
Towards a Cooperative Traffic Network Editor
}

\author{
José L. F. Pereira ${ }^{*}$, Rosaldo J. F. Rossetti ${ }^{\dagger}$, Eugénio C. Oliveira ${ }^{\dagger}$ \\ Artificial Intelligence and Computer Science Lab (LIACC) \\ ${ }^{\dagger}$ Department of Informatics Engineering \\ *Department of Electrical and Computer Engineering \\ Faculty of Engineering, University of Porto (FEUP) \\ Rua Dr. Roberto Frias, S/N • 4200-465 Porto • Portugal \\ \{ee06201, rossetti, eco\}@fe.up.pt
}

\begin{abstract}
In this paper we explore the potential benefits of concepts such as visual interactive modelling and simulation to devise and implement a cooperative network editor embedded in a collaborative environment for transport analysis. Traditional approaches lack adequate means to foster integrated analyses of transport systems either because they are strict in terms of purpose or because they do not allow multiple users to dynamically interact on the same description of a model. The use of a common geographical data model of the application domain promotes the means for different experts to interact seamlessly in a collaborative environment.
\end{abstract}

Keywords: cooperative modelling, cooperative simulation, visualisation for multiple users, cooperative traffic and transportation engineering.

\section{Introduction}

Intelligent Transportation Systems (ITS) have recently gained a prominent role in everyone's daily lives. In fact, the information and communication infrastructure currently available is promising a great revolution and the Future Urban Transport (FUT) systems seem more feasible. ITS-based technologies toward FUT bring the user to a central spot and strive to address many issues concerning mobility and the quality of life in highly populated areas. These issues are rather related to the qualitative assessment by users with different perceptual abilities, which suggest a number of new performance measures that need to be accounted for and assessed through powerful and expressive tools [2]. In fact, transport problems have been traditionally tackled from different perspectives. Therefore, an imperative evolution, required for the sake of productivity, is the integration of tools, models and analysis methods into a common simulation framework [3]. More recently, however, cooperation mechanisms have been identified as imperative for such integration [1].

This paper follows up the work started in [1] and suggests the concepts of visual interactive modelling (VIM) and simulation (VIS) as crucial elements to foster the implementation of a cooperative network editor embedded in a collaborative transport analysis environment. In fact, the traffic network is the basis for virtually all transport models as it is the essence of the urban mobility infrastructure. However, different tools, with different purposes, have adopted different data structures and models to 
represent some concepts that transverse all subfields related to transport engineering and urban planning. Then, allowing experts with different perspectives to interact cooperatively onto a common urban model is a key ingredient to foster the so desired integrated perspective of transport analysis.

\section{Cooperative VIS \& VIM}

The manipulation and visualisation through graphical representations of any attribute allow us to qualitatively evaluate the state of a system in an intuitive and quick way. Thus using visual interactive features in any simulation framework has become imperative, covering every step within the user interaction process. The visual interactive simulation (VIS) concept implies the use of interactive visual features aiming at steering the simulation process and allowing intermediate data visualisation and model parameters control. Such a concept has been initially approached by Hurrion [5] in the mid 70's, albeit its popularisation was effectively achieved in the 80's. In a similar way, aiming at increasing productivity during first stages of the simulation process through visual interactive features, e.g. model construction and data preparation, the concept of visual interactive modelling (VIM) was defined to cover all phases of the simulation project [4].

Considering the possibility of directly interacting with model's parameters, Marshall [6] identified three basic types of user-simulation interactions: post-processing, tracking and steering. Post-processing allows the user to graphically visualise simulation results only after an experiment has been concluded, thus no interaction is actually possible. During tracking, visualisation occurs while the simulation experiment is running. However, no interaction is possible as well, albeit the user can still interrupt the process. In the latter case, the user can steer the simulation process and interact with the model in runtime. Users can modify model parameters and entity attributes as the process evolves, and immediately visualise the results of such interactions.

In this work, we extend the boundaries of VIS and VIM beyond the integration of both concepts as suggested in [4]. In fact, issues related to the cooperative handling of simulation models in a way that different users can interact at the same time and still perceive how others' interaction will affect their simulation results remain to be addressed. Thus allowing post-processing, tracking and steering on a cooperative basis arises as an interesting and challenging research subject.

\section{The Environment Architecture}

Bearing the above perspective in mind, we can create an architecture based on whitebox components capable of dynamically adapting themselves and interacting with each other as the current situation requires. Our approach is represented in the collaborative environment depicted in Fig. 1.

As most information in our application domain is geographically referenced, we use a specialised geographical information system (GIS) as a facilitator of the communication among multiple areas of interest. The GIS model will soon be extended into a geographical ontology, serving not only as a persistence and integration mecha- 
nism but also as the interface between different traffic network analyses tools aimed at diverse purposes. Thus practitioners from different backgrounds, e.g. traffic monitoring and control, urban planning, environment and pollution, public transportation, and many others, would have an environment where they could seamlessly interact and collaborate. In order to accomplish this, we started by featuring the GIS database with a proper meta-data for simple and compatible conversion between greatly used data formats, making it easy to migrate them into this approach.

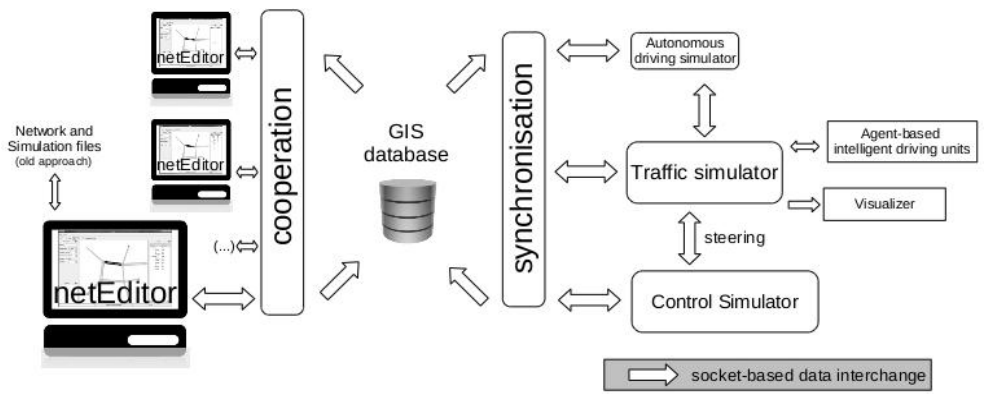

Fig. 1 A collaborative traffic analysis environment with cooperative modelling abilities

The authentication mechanism intrinsic to most database management systems as well as access control policies facilitate controlling users who can edit, delete, create and read objects of the network model, whereas a log of every modification made is kept for diverse purposes. The proposed database-centric approach bridges two significant components, namely the cooperation and the synchronization layers. These two components distinguish between the modelling phase (on the left of Fig. 1) and the analysis phase (on the right of Fig. 1), where users can run different analysis tools from complex simulation to simple visualisation of results and objects' attributes.

The cooperation layer comprises all the edition tools needed to perform maintenance of the database. Different applications, such as instances of the traffic network editor (netEditor) we have implemented or a traffic signal editor can be easily attached to this layer, and the interaction with the database can be made in two ways, namely synchronously or asynchronously. In the former case, editors send all the updates to the database as quickly as the socket connection permits, allowing various users to cooperate on the same sub-network model as if they were working physically together. In the latter case, the user can lock a specific part of the network (only this user will have access to the selected sub-network model, thus minimizing the risk of conflicts) and commit all changes after manipulating it. After this, all applications connected to the database are updated, making changes noticeable to everyone.

The synchronization layer contains all the aspects related to the analysis processes, naturally including any simulation tools. These tools can either keep an on-line connection to the database while running any analysis processes or perform such processes on an off-line basis. In the former case, any modification to the database through the cooperation layer will directly affect the analysis processes running on the other layer. On the contrary, if analyses are run off-line a sub-network model image is kept on the right side, avoiding interferences from processes running on the left side.

According to the purpose of the analysis tool, a hole network can be simulated or just a part of it. This allows us to migrate across different granularity scales dynami- 
cally, favouring different simulation perspectives (e.g. micro, meso and macroscopic simulation). Fig. 2 depicts the cooperative network editor attached to the cooperative layer, whereas a microscopic simulator is running on the synchronisation layer.

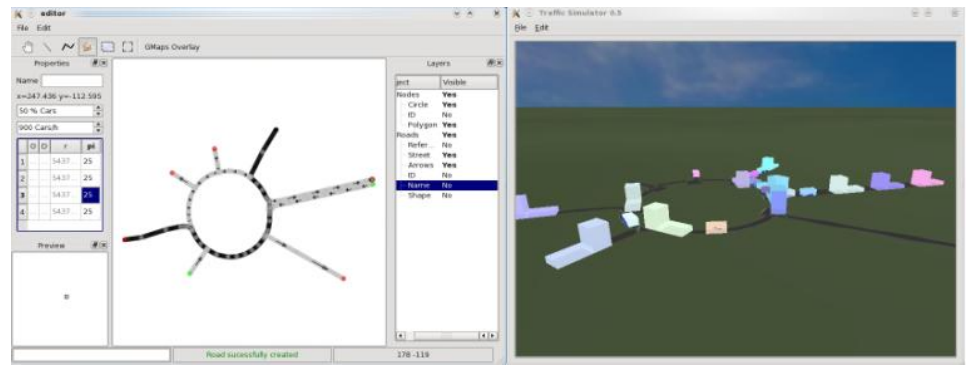

Fig. 2 The cooperative netEditor and a microscopic traffic simulator

\section{Conclusions}

Integrating different perspectives in transport analysis is a long-term goal that the scientific community has been striving to accomplish. Whereas first attempts targeted fully integrated stand-alone simulation frameworks, distributed environments that today underlie most computer systems favours cooperation on a distributed basis. In this work we turn back to the concepts of VIM and VIS, considering their potential application to improve cooperation on a distributed environment to feature a traffic network editor with characteristics such as post-processing, tracking and steering. The proposed cooperative architecture is an extension to the interaction mechanisms underlying the true concept behind the MAS-Ter Lab platform [1], while the implemented prototype demonstrated the viability of the approach with some graphical and interactive features tested. The improvement of the domain ontology to support dynamic and flexible interaction among different experts is the next step in this research.

\section{References}

1. Ferreira, P.A.F., E.F. Esteves, R.J.F. Rossetti, E.C. Oliveira (2008) A Cooperative Simulation Framework for Traffic and Transportation Engineering. In Cooperative Design, Visualization, and Engineering, CDVE (LNCS 5220). pp.89-97.

2. Klefstad, R., Y. Zhang, M. Lai, R. Jayakrishnan, R. Lavanya (2005) A Scalable, Synchronized, and Distributed Framework for Large-Scale Microscopic Traffic Simulation. In IEEE Conference on Intelligent Transportation Systems. pp.813-818.

3. Rossetti, R.J.F., S. Bampi (1999) A Software Environment to Integrate Urban Traffic Simulation Tasks. Journal of Geographic Inf. and Decision Analysis, 3(1):56-63.

4. Wagner, P.R., C.M.D.S. Freitas, F.R. Wagner (1996) A new paradigm for visual interactive modelling and simulation. In European Simulation Symposium. pp.142-145.

5. Hurrion, R.D. (1976) The design, use, and required facilities of an interactive visual computer simulation language to explore production planning problem. London: University of London, Ph.D. Thesis.

6. Marshall, R., J. Kempf, S. Dyer, C.-C. Yen (1990) Visualization methods and simulation steering for a 3D turbulence model of lake Erie. Computer Graphics 24(2):89-97. 